\title{
Dynamics of Electrically Driven Martensitic Phase Transitions in Fe Nanoislands
}

\author{
Lukas Gerhard, ${ }^{1,2}$ Rien J. H. Wesselink, ${ }^{3}$ Sergej Ostanin, ${ }^{4}$ Arthur Ernst, ${ }^{4,5}$ and Wulf Wulfhekel ${ }^{1,2}$ \\ ${ }^{1}$ Physikalisches Institut, Karlsruhe Institute of Technology, 76131 Karlsruhe, Germany \\ ${ }^{2}$ Institut für Nanotechnologie, Karlsruhe Institute of Technology, 76344 Eggenstein-Leopoldshafen, Germany \\ ${ }^{3}$ Faculty of Science and Technology and MESA + Institute for Nanotechnology, \\ University of Twente, 7500AE Enschede, The Netherlands \\ ${ }^{4}$ Max-Planck-Institut für Mikrostrukturphysik, Weinberg 2, 06120 Halle, Germany \\ ${ }^{5}$ Wilhelm-Ostwald-Institut für Physikalische und Theoretische Chemie, Universität Leipzig, Linnéstraße 2, 04103 Leipzig, Germany
}

(Received 12 April 2013; revised manuscript received 11 September 2013; published 14 October 2013)

\begin{abstract}
Magnetoelectric coupling has attracted interest due to its potential to write magnetic information with electric fields. In the model system of Fe islands on $\mathrm{Cu}(111)$, electric fields can induce martensitic phase transitions between ferromagnetic body-centered cubic and antiferromagnetic face-centered cubic phases. Here, we present a detailed study of the dynamics and energetics of the phase transition in the electric field of the junction of a scanning tunneling microscope. Statistical measurements allow us to reveal the influence of both the electric field and the crystallographic strain on the energy landscape of the two competing phases.
\end{abstract}

DOI: 10.1103/PhysRevLett.111.167601

PACS numbers: 77.55.Nv, 68.37.Ef, 71.15. $-\mathrm{m}, 75.75 .-\mathrm{c}$

The possibility of using an electric field to influence magnetism in metallic nanostructures [1-3] gives new prospects towards the creation of high-density metallic memories [4-8].

As a direct link between the sign of the applied electric field and the magnetization direction of a ferromagnet is impossible [6], an electric-field-induced switching between bistable magnetic states of a metal was not observed until very recently [9-13]. One solution is to exploit the influence of an electric field on the lattice of a metallic surface [14] and thereby to influence the magnetic order. It has been shown that the surface of Fe nanoislands is a suitable model system that shows strong lattice-mediated magnetoelectric coupling (MEC) via a martensitic phase transition [9].

$\mathrm{On}$ a $\mathrm{Cu}(111)$ surface, in the low coverage regime below two monolayers (ML), Fe islands of 2 ML thickness exhibit a coexistence of two crystallographic phases [15]: a dominant face-centered cubic (fcc) phase in the center of the islands and a narrow body-centered cubic (bcc)-like phase at the rime of the islands [9]. At coverages above $2 \mathrm{ML}$, the evolving film undergoes a full phase transition to bcc Fe [15]. Early magneto-optic Kerr effect (MOKE) measurements [16] revealed that at coverages below 2 ML the Fe film shows a low magnetic moment whereas at coverages above $2 \mathrm{ML}$ a phase with the full magnetic moment evolves. This can be explained by the magnetic order of the fcc and bcc phases. Whereas the bcc phase is ferromagnetic with its full magnetic moment, the fcc phase is layerwise antiferromagnetic [9]. The bottom $\mathrm{Fe}$ layer shows a magnetic moment of $2.2 \mu_{B}$ whereas the top layer shows only $1.9 \mu_{B}$ [17]. This explains the low magnetic moment observed with MOKE.

In our recent paper [9], we confirmed the results of the Varga group at $5 \mathrm{~K}$, i.e., the coexistence of fcc and bcc phases of $\mathrm{Fe}$ in individual islands. Furthermore, we performed spin-polarized scanning tunneling microscopy (STM) on both phases. Within the two phases, no spin contrast could be observed. This situation complies with ferromagnetic and with layerwise antiferromagnetic order. To distinguish between these two, we recorded tunneling spectroscopy of both phases and compared it with ab initio calculated local density of states (LDOS) of the two magnetic orders in the two phases. Whereas the calculated LDOS of antiferromagnetically ordered bcc films and ferromagnetically ordered fcc films deviate significantly from the measured LDOS, the measured LDOS fitted very nicely to the calculated LDOS for ferromagnetic bcc and antiferromagnetic fcc phases [9] in full agreement with previous work. A reversible martensitic phase transition between these two phases can be induced by the applied electric field in the junction of an STM [9]. It was shown experimentally and theoretically that the two bistable states can be switched by a high electric field and that depending on the polarity of the field, either the bcc or the fcc phase is stabilized. This electric-field-induced structural change has been shown by both atomically resolved STM pictures and the change of the experimental LDOS. This martensitic phase transition was uniquely observed on bilayer islands, as it delicately depends on the balance between the two phases that is determined by the interfacial strain. A rigorous understanding of the dynamic processes and the behavior at intermediate electric fields is, however, missing.

We here report on the switching dynamics of the transition between the two phases as a function of the electric field and the local strain. A simple model of the energy landscape gives insight into the switching process and allows us to measure both the energy difference between 
the two phases and the barrier heights separating the two as functions of the applied electric field and local strain of the Fe lattice. Experimental results are compared with state of the art $a b$ initio calculations.

The basic switching experiments were carried out as follows. By deposition of a submonolayer amount of $\mathrm{Fe}$ on a $\mathrm{Cu}(111)$ substrate by molecular beam epitaxy at room temperature, locally $2 \mathrm{ML}$ high Fe nanoislands were grown. These samples were studied in a low-temperature STM at $\approx 5 \mathrm{~K}$. The preparation of the sample and all measurements were carried out in ultrahigh vacuum conditions. In order to get insight into the dynamics of the phase transition, we positioned the STM tip above the area of interest on the Fe island and applied a constant electric field. Then, we recorded the differential conductance signal $(d I / d V)$ at a fixed tip position as a function of time. In the case that both crystallographic states are at similar energy levels and the barrier in between is low enough to allow thermally activated transitions, individual transitions can be observed as telegraph noise in the $d I / d V$ signal [see Fig. 1(a)]. The two phases display different local density of states and thus different $d I / d V$ signals [9]. The population of the two phases is given by Boltzmann statistics; the ratio of the two is directly linked to their energy difference

$$
\frac{N_{\mathrm{bcc}}}{N_{\mathrm{fcc}}}=e^{-\left(E_{\mathrm{bcc}}-E_{\mathrm{fcc}}\right) / k_{B} T}
$$

(a)

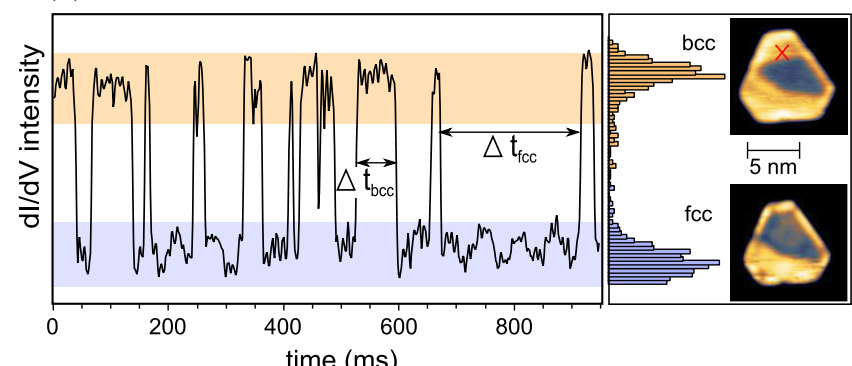

(b)

(c)
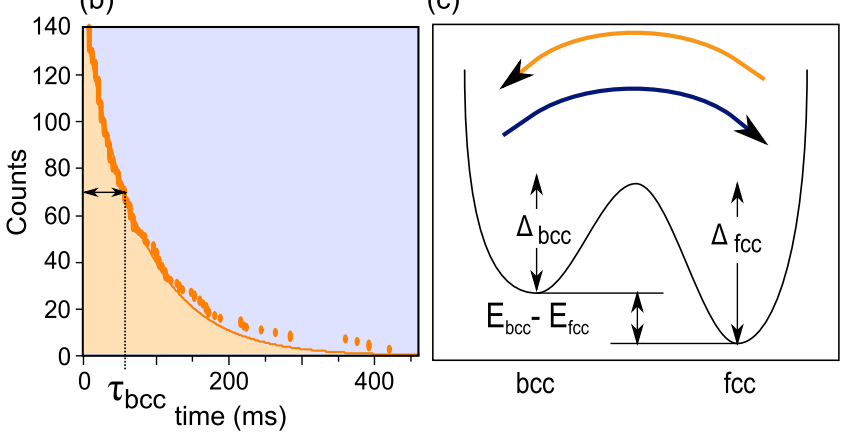

FIG. 1 (color online). (a) $d I / d V$ signal at an applied electric field of $0.63 \mathrm{GV} / \mathrm{m}$ recorded at the position indicated by the red cross in the inset (topographic snapshots). The histogram on the right side reflects the distribution of the $d I / d V$ signal showing two distinct peaks for bcc and fcc states. (b) The decay of the bcc state (orange squares) can be fitted with an exponential decay (solid orange line). (c) Energy diagram of the bistable switching between bcc and fcc. with $k_{B}$ the Boltzmann constant and $E_{\mathrm{fcc}}$ and $E_{\mathrm{bcc}}$ the energies of the fcc and bcc states, respectively.

In order to determine the population of each state we first identify the two phases by their $d I / d V$ signal using the colored intervals in Fig. 1(a) as discriminators. From Eq. (1), we obtain an energy difference of $0.05 \mathrm{meV}$ slightly favoring the fcc state at this particular electric field and at this position of the island. As individual switching events can be resolved, the data contain not only information on the energy difference but also on the involved barriers. From the individual residence times, an average lifetime can be extracted. For this, we plot the $N$ extracted residence times $\Delta t_{\mathrm{bcc}}$ as a decay of a population of $N$ [orange marks in Fig. 1(b)]. The decay is clearly exponential. From a fit we can extract the lifetime of the bcc state $\left(\tau_{\text {bcc }}=60 \mathrm{~ms}\right)$. This exponential decay is indicative of an activated transition, and the system can be modeled by an energy landscape with the fcc and the bcc states as local minima [see Fig. 1(c)]. The lifetime $\tau_{\mathrm{bcc}}$ of the bcc state is related to the barrier height $\Delta_{\text {bcc }}$ by transition-state theory $[18,19]$

$$
\tau_{\mathrm{bcc}}^{-1}=\nu_{0} e^{-\left[\Delta_{\mathrm{bcc}} /\left(k_{B} T\right)\right]},
$$

where $\nu_{0}$ represents the attempt frequency for the transition, which is related to thermal phonons (at $5 \mathrm{~K}$ ) of typical frequencies of the order of $\nu=k_{B} T / h=10^{11} \mathrm{~Hz}$. The exact frequency is not known, such that only trends in the barrier height can be investigated. An absolute determination would require measurements over a large temperature range, which is not feasible in our setup. In this particular example of $\left(\tau_{\mathrm{bcc}}=60 \mathrm{~ms}\right)$, a barrier height of $9.4 \mathrm{meV}$ was estimated. In the same way, we determined the lifetime and barrier height of the fcc state. These characteristic energies depend on the electric field but also on the local lattice strain. The latter varies within the island due to the partial relaxation at the island edges.

To determine the dynamics of the martensitic phase transition as a function of the applied electric field, the tip was positioned above the sample at a fixed position and the bias voltage was ramped slowly while the $d I / d V$ signal was recorded. Figure 2(a) depicts a typical resulting curve that reflects both the electric field dependence and the time trace of the signal. The large scale change of the differential conductance reflects the bias-dependent LDOS. Besides, two different states of high and low differential conductance are found, similar to the case of Fig. 1(a). Clearly, the lifetimes and the population of the two states vary with the applied electric field. At discrete electric fields, long time traces of the $d I / d V$ signals were recorded to obtain better statistics. Each measurement was analyzed as described above, and the resulting values for the lifetimes were calculated [cf. Figs. 2(b) and 2(c)]. At low electric fields $(E=0.1 \mathrm{GV} / \mathrm{m})$, no switches were observed within the recording time, indicating lifetimes significantly greater than $300 \mathrm{~s}$ for fcc and bcc states. Only at slightly larger 
electric fields of $E=0.13 \mathrm{GV} / \mathrm{m}$ does switching begin. Thus for low electric fields (dark gray region), lifetimes are long, implying that the energy barriers are hardly overcome by the thermal fluctuations at $5 \mathrm{~K}$. Note that the lifetimes of both states are of the same order of magnitude. For medium-high electric fields (light gray regions), the lifetimes are reduced by orders of magnitude; i.e., the electric field facilitates switching by reducing the energy barriers. Nevertheless, the fcc and bcc states are still equally populated, and their lifetimes are of the same order. Only at high electric fields ( \pm 0.3 to $\pm 3 \mathrm{GV} / \mathrm{m}$ ) does a clear asymmetry between the lifetimes emerge. Thus, with rising magnitude of the electric field, first the barriers are lowered and then an imbalance in the energy of the states is established. This is well reflected in Fig. 2(d), where we plot the energy difference between the bcc and fcc states [see Eq. (1)]. At low and intermediate fields, the difference vanishes within the

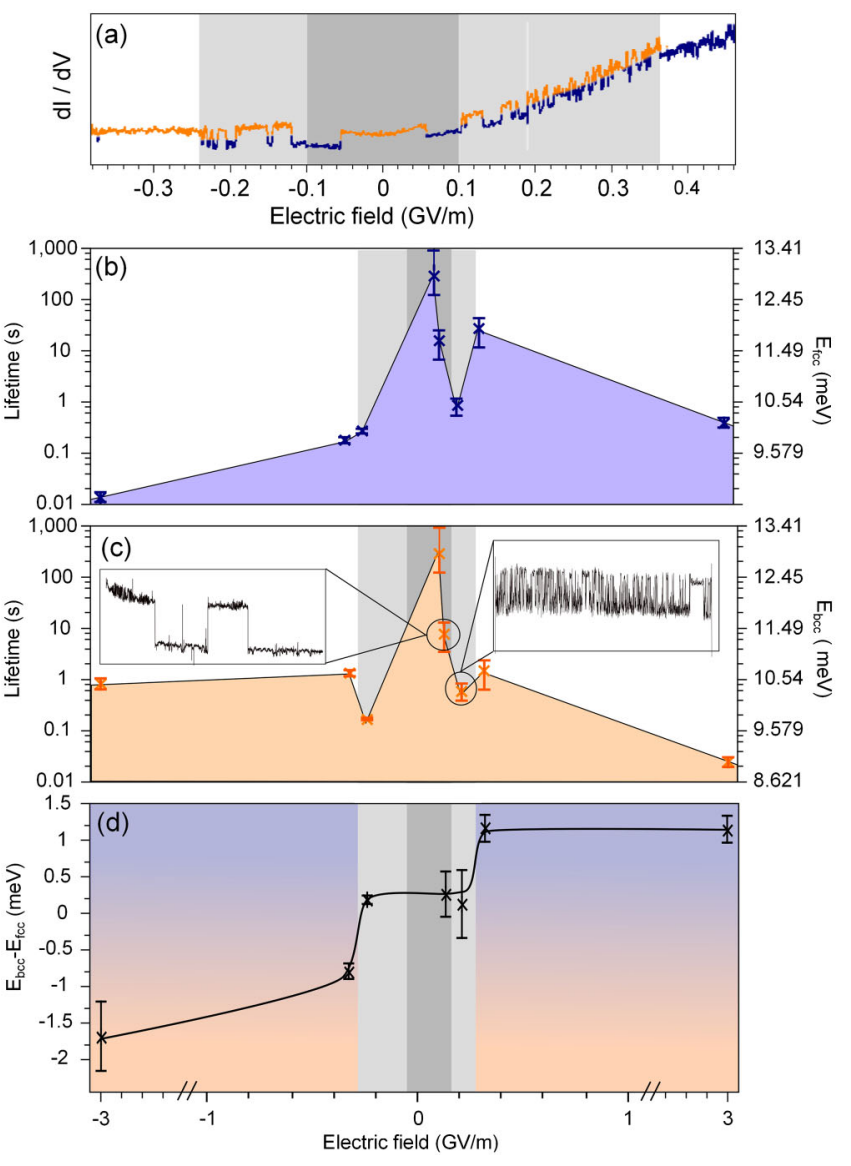

FIG. 2 (color online). (a) Typical $d I / d V$ signal as function of time and applied electric field. (b) Lifetimes and energy barrier of the fcc state. (c) Lifetimes and energy barrier of the bcc state. (d) Energy differences between the two phases at different electric fields. The connecting lines are a guide to the eye. The range of low electric fields is indicated by the vertical dark gray area; medium-high electric fields are indicated by vertical light gray areas. The inset in (c) shows two representative time traces recorded at the indicated electric fields. statistical error, whereas at high positive (negative) fields, the fcc (bcc) state is favored by $1-2 \mathrm{meV}$ per atom.

Thus, the analysis of the switching dynamics confirms our prior observations $[9,20]$ that by the application of large electric fields, either the bcc or fcc phase can be induced. The analysis of the dynamics of the martensitic phase transition, however, allows us to give quantitative energy differences and energy barriers as functions of the electric field. The barriers vary between 9 and $13 \mathrm{meV} /$ atom, and the energy differences vary between +1.5 and $-1.5 \mathrm{meV} /$ atom. These are significantly lower than those based on the ab initio theory presented in our recent publication [9].

In order to lift this discrepancy, more realistic firstprinciples calculations of MEC in Fe were carried out, approximating finite islands by two monolayers of $\mathrm{Fe}$ on $\mathrm{Cu}(111)$ as done in our original work [see Ref. [9]]. The calculations were performed using a self-consistent Green function method, specially designed for layered semiinfinite systems [21]. A high electric field was simulated by a plate capacitor placed in the vacuum at a certain distance above the Fe film. In the calculations, vertical relaxations were taken into account as well as the lateral movement of the atoms from the threefold fcc positions to the bridge positions of the bcc configuration. We, however, also included the lateral deformation in the transition from the fcc to the bcc unit cell. The last considerations, especially, play an important role for the barrier heights. On top of these considerations, the local strain in the islands especially near the island edge is unknown. To model the local strain, the lattice parameter of the $\mathrm{Cu}$ substrate below the 2 ML Fe film was slightly varied, and the role of this strain on the energy balance in an electric field was investigated. The results of our simulations are presented in Fig. 3, which show the relative total energy per unit cell as a function of lateral displacement of the atoms of the top Fe layer due to the martensitic phase transition. Layerwise antiferromagnetic order is shown in blue (dark gray), and ferromagnetic order is shown in orange (light gray). For all three cases (with a $\mathrm{Cu}$ lattice constant ranging from $98 \%$ to $102 \%$ of the bulk value), we find local minima for both fcc and bcc stacking and a decisive influence of the electric field (see Fig. 3): negative fields (a) favor the bcc stacking whereas positive fields (c) favor the fcc stacking in full agreement with the experiment. The energy differences between the local minima for the case of zero electric fields are in the range of 1 to $3 \mathrm{meV} /$ atom [see Fig. 3(b)], which is of the same order of magnitude as that in the experiments. In case of high fields, however, the energy differences are higher than those in the experiment. This can be explained by the discrepancy in the lateral dimensions. While the calculations were performed for infinite layers with a fixed lattice constant, the experiments deal with small islands in which the strain in the $\mathrm{Fe}$ partially relaxes at the borders of the islands. Furthermore, 


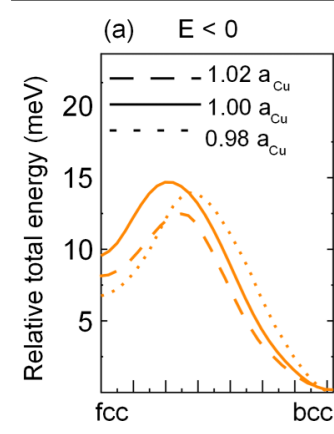

(b) $E=0$

(c) $E>0$
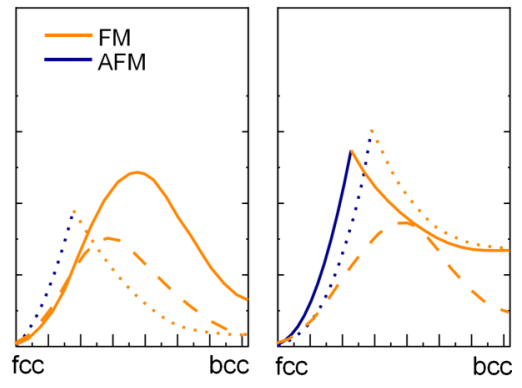

Lateral displacement

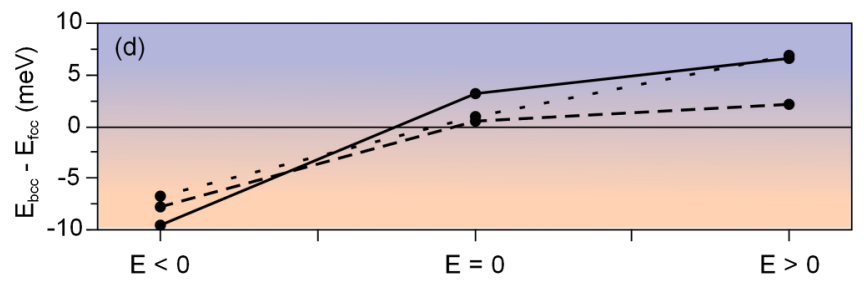

FIG. 3 (color online). Ab initio calculations for the energy landscape of the fcc to bcc transition in (a) negative, (b) zero, and (c) positive applied electric field and slightly different lattice constants of the $\mathrm{Cu}$ substrate. Blue (dark gray) lines indicate layerwise antiferromagnetic order, and orange (light gray) lines indicate ferromagnetic order. (d) Energy differences between the two states for the three different values of the electric field. The connecting lines are guides to the eye.

density functional theory does not describe the surface charge layer fully correctly.

In the experimental islands, the difference in the lattice constants of $\mathrm{Fe}$ and the $\mathrm{Cu}(111)$ substrate leads to strain that is not uniform but depends on position. In the center of large islands, the $\mathrm{Fe}$ atoms experience the highest strain and adopt the fcc stacking. At the edges, the atoms are able to partially relax and adopt the bcc structure. Close to the domain boundary, the strain is intermediate. This difference in strain is responsible for the coexistence of the two crystallographic phases and is expected to be of crucial importance for the phase transition. Hence, MEC in $2 \mathrm{ML}$ Fe can be studied at different strains by measuring at different positions on the island. In a second experiment, we therefore measure the electric-field-dependent switching parameters with high lateral resolution of the position of the tip above the island. First, a topographic image of an Fe island at low electric field was acquired. This gives the native distribution of the two phases and thus reflects the effect of lattice strain (see inset in Fig. 4). We recorded time traces on three different areas [rectangles indicated in red (gray) in the inset of Fig. 4] that correspond to different strain in the Fe film. For each strain value, the lifetimes of the two states were evaluated at four different electric fields (see Fig. 4). This is the expected behavior of bulk Fe [22] and is partly reproduced by our calculations, as the antiferromagnetic fcc is the ground state only for the smaller lattice constants [see blue (dark gray) lines in Fig. 3]. It can be seen in all curves that, similar to Fig. 2, the lifetimes

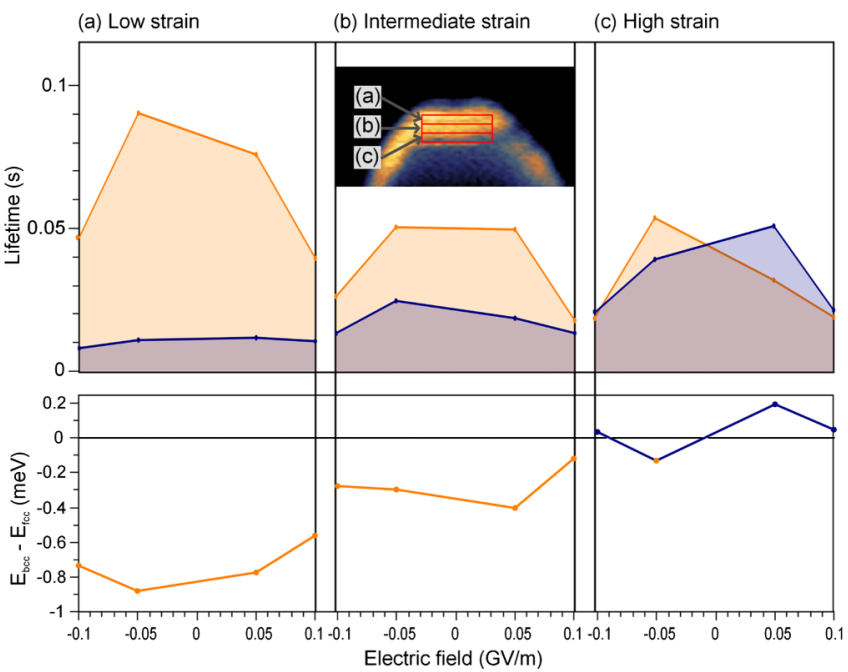

FIG. 4 (color online). Strain dependence of switching at different electric fields. The inset depicts the three areas on which the measurements were performed. Lifetimes (upper parts) and the energy differences (lower parts) at (a) low strain, (b) intermediate strain, and (c) high strain.

decrease with increasing the electric field from a low to an intermediate (positive or negative) value. Furthermore, the ratio of the lifetimes, which reflects the energy difference between the two states, does not vary significantly within this electric field range [see lower parts in Figs. 4(a)-4(c)]. However, the energy difference between the two states varies strongly with strain: at lower strain, the ferromagnetic bcc phase is clearly favored [see Fig. 4(a)] whereas the higher strain present in the lower row favors the antiferromagnetic fcc phase [see Fig. 4(c)]. This is the expected behavior of bulk Fe [22] and is partly reproduced by our calculations, as the antiferromagnetic fcc is the ground state only for the smaller lattice constants [see blue lines in Fig. 3]. This shows that the energy balance between the two phases delicately depends on the strain in the island imposed by the lattice mismatch: a change of position by less than $1 \mathrm{~nm}$ changes the energy balance by almost $1 \mathrm{meV}$.

In conclusion, we have shown that by using scanning tunneling microscopy it is possible to study the energy landscape of a martensitic phase transition triggered by electric fields on the nanoscale. We disentangled the complex influence of electric field and lattice strain on the dynamic behavior of the antiferromagnetic fcc to ferromagnetic bcc transition in $2 \mathrm{ML} \mathrm{Fe} / \mathrm{Cu}(111)$. Both the energy balance of the two phases and the barrier between them delicately depend on lateral strain due to the lattice mismatch with the $\mathrm{Cu}$ substrate and the vertical strain externally induced by the electric field. Ab initio calculations fully reproduce the influence of the electric field and qualitatively agree with the experimentally determined energies. This provides the possibility to fine tune the dynamics of MEC-induced phase transitions in Fe films 
by varying the lattice constant of the substrate or the applied electric field.

We acknowledge funding by the German Research Foundation (DFG Grants No. ER 340/4-1 and No. WU 349/8-1).

[1] M. Weisheit, S. Fähler, A. Marty, Y. Souche, C. Poinsignon, and D. Givord, Science 315, 349 (2007).

[2] D. Chiba, S. Fukami, K. Shimamura, N. Ishiwata, K. Kobayashi, and T. Ono, Nat. Mater. 10, 853 (2011).

[3] U. Bauer, M. Przybylski, J. Kirschner, and G. Beach, Nano Lett. 12, 1437 (2012).

[4] R. Ramesh, Nat. Nanotechnol. 5, 761 (2010).

[5] W. Eerenstein, N.D. Mathur, and J.F. Scott, Nature (London) 442, 759 (2006).

[6] N. A. Spaldin and M. Fiebig, Science 309, 391 (2005).

[7] T. Lottermoser, T. Lonkai, U. Amann, D. Hohlwein, J. Ihringer, and M. Fiebig, Nature (London) 430, 541 (2004).

[8] N. N. Negulyaev, V.S. Stepanyuk, W. Hergert, and J. Kirschner, Phys. Rev. Lett. 106, 037202 (2011).

[9] L. Gerhard, T. K. Yamada, T. Balashov, A. F. Takacs, R. J. H. Wesselink, M. Däne, M. Fechner, S. Ostanin, A. Ernst, I. Mertig et al., Nat. Nanotechnol. 5, 792 (2010).
[10] Y. Shiota, T. Nozaki, F. Bonell, S. Murakami, T. Shinjo, and Y. Suzuki, Nat. Mater. 11, 39 (2012).

[11] W.-G. Wang, M. Li, S. Hageman, and C. Chien, Nat. Mater. 11, 64 (2012).

[12] E. Tsymbal, Nat. Mater. 11, 12 (2012).

[13] S. Kanai, M. Yamanouchi, S. Ikeda, Y. Nakatani, F. Matsukura, and H. Ohno, Appl. Phys. Lett. 101, 122403 (2012).

[14] J. Weissmüller, R. N. Viswanath, D. Kramer, P. Zimmer, R. Würschum, and H. Gleiter, Science 300, 312 (2003).

[15] A. Biedermann, W. Rupp, M. Schmid, and P. Varga, Phys. Rev. B 73, 165418 (2006).

[16] P. Ohresser, J. Shen, J. Barthel, and M. Zheng, Ch. V. Mohan, M. Klaua, and J. Kirschner, Phys. Rev. B 59, 3696 (1999).

[17] Arthur Ernst (unpublished).

[18] H. Eyring, J. Chem. Phys. 3, 63 (1935).

[19] H. Ibach, Physics of Surfaces and Interfaces (Springer, Berlin, 2006).

[20] T. K. Yamada, L. Gerhard, T. Balashov, A. F. Takács, R. J.H. Wesselink, and W. Wulfhekel, Jpn. J. Appl. Phys. 50, 08LA03 (2011).

[21] M. Lüders, A. Ernst, W. M. Temmerman, Z. Szotek, and P. J. Durham, J. Phys. Condens. Matter 13, 8587 (2001).

[22] V. L. Moruzzi, P. M. Marcus, K. Schwarz, and P. Mohn, Phys. Rev. B 34, 1784 (1986). 